\title{
Metaplastic breast carcinomas are enriched in markers of tumor-initiating cells and epithelial to mesenchymal transition
}

\author{
Yanhong Zhang ${ }^{1,2,3}$, Kathy A Toy ${ }^{1,2}$ and Celina G Kleer ${ }^{1,2}$ \\ ${ }^{1}$ Department of Pathology, University of Michigan Medical School, Ann Arbor, MI, USA and \\ ${ }^{2}$ Comprehensive Cancer Center, University of Michigan Medical School, Ann Arbor, MI, USA
}

\begin{abstract}
Metaplastic breast carcinomas constitute a distinct aggressive form of invasive breast cancer with histological evidence of epithelial to mesenchymal transition toward spindle, chondroid, or osseous cell types. During tumorigenesis, epithelial to mesenchymal transition promotes invasion and metastasis and has been linked to the presence of stem cells. We hypothesized that metaplastic carcinomas may express epithelial to mesenchymal transition markers and may be enriched in tumor-initiating cells specifically in the non-glandular metaplastic elements. In 27 primary metaplastic carcinomas of the breast we tested the expression of epithelial to mesenchymal transition inducers ZEB1 and E-cadherin and the presence of tumor-initiating cells by using aldehyde dehydrogenase-1 (ALDH-1) and $\mathrm{CD}_{4}{ }^{+} / \mathrm{CD}^{-1 / \text { low }}$ immunohistochemistry. Of the 27 metaplastic carcinomas, $20(74 \%)$ had squamous and/or spindle areas and $7(26 \%)$ had heterologous elements $(6$ chondroid and 1 osseous). ALDH-1-positive and $\mathrm{CD} 44^{+} / \mathrm{CD}^{2} 4^{- \text {llow }}$-expressing cells were detected in the non-glandular metaplastic components (Fisher's exact, $\boldsymbol{P}=\mathbf{0 . 0 0 1 7}$ ). E-cadherin expression was reduced or absent (aberrant) in all metaplastic components whereas it was normal in the glandular areas. On the contrary, overexpression of ZEB1 was detected in $41 \%$ (11 of 27) of the non-glandular, metaplastic components, and in none of the glandular areas. The presence of tumor-initiating cells, aberrant E-cadherin, and ZEB1 upregulation was associated in over $90 \%$ of the spindle areas and heterologous elements $\left(\chi^{2}\right.$ test, $\left.P<0.05\right)$. We provide first in situ evidence that epithelial to mesenchymal transition inducers and tumor-initiating cells are present specifically in the nonglandular components of metaplastic carcinomas.
\end{abstract}

Modern Pathology (2012) 25, 178-184; doi:10.1038/modpathol.2011.167; published online 11 November 2011

Keywords: aldehyde dehydrogenase; CCN6; epithelial to mesenchymal transition; E-cadherin; metaplastic breast cancer; WISP3; ZEB

Metaplastic mammary carcinomas are a histologically heterogeneous and unique group of tumors defined by the presence of glandular and nonglandular component. ${ }^{1}$ The non-glandular component results most of the times from mesenchymal differentiation and includes cells with spindle, osseous, or cartilaginous features. Metaplastic carcinomas are almost invariably negative for hormone

Correspondence: Dr CG Kleer, Department of Pathology, University of Michigan Medical School, 4217 Comprehensive Cancer Center, 1500 E Medical Center Dr, Ann Arbor, MI 48109, USA. E-mail: kleer@umich.edu

${ }^{3}$ Current address: Department of Pathology, University of California Davis Medical Center.

This work was presented at the 100th Meeting of the United States and Canadian Academy of Pathology, 2011.

Received 13 July 2011; revised 8 September 2011; accepted 8 September 2011; published online 11 November 2011 receptors and do not exhibit HER-2/neu overexpression. ${ }^{1,2}$ Metaplastic carcinomas have a high rate of systemic metastasis and, with the exception of the rare pure low-grade spindle cell carcinoma, they have a discouraging prognosis. ${ }^{1,3}$ Understanding the pathobiology of metaplastic carcinomas is critical to develop new and effective treatments.

Metaplasia is the reversible change in which one adult cell type is replaced by another adult cell type, and it is currently thought to arise from genetic reprogramming of stem cells. ${ }^{4} \mathrm{~A}$ mechanism by which cells may undergo metaplasia during tumorigenesis is through an epithelial to mesenchymal transition. Through this process, tumor cells acquire molecular and phenotypic changes resulting in spindle morphology and dysfunctional cell-cell adhesion, leading to invasion and metastasis. ${ }^{5-7}$ Recently, epithelial to mesenchymal transition was 
shown to induce the acquisition of breast cancer stem cell properties. ${ }^{8,9}$ One of the most frequent molecular alterations in the epithelial to mesenchymal transition process is the loss of membranous E-cadherin, which facilitates cell detachment. ${ }^{5,6}$ The E-cadherin transcriptional regulator ZEB1 is highly expressed in breast cancer cell lines with an invasive and metastatic phenotype compared with non-invasive cell lines. ${ }^{10}$ Although the ability of ZEB1 to repress E-cadherin has been shown at the molecular level, ${ }^{11-13}$ it has not been well documented in vivo and has not been examined in metaplastic carcinoma.

Recent studies of metaplastic breast carcinomas revealed a prominent epithelial to mesenchymal transition signature as well as enrichment for inducers of tumor stem cell characteristics. ${ }^{14-17}$ However, whether cells undergoing an epithelial to mesenchymal transition have characteristics of tumor-initiating cells in human breast tissue samples is not known. In an effort to translate these mechanistic and gene profiling data into clinically relevant observations in human metaplastic carcinomas, we tested the hypothesis that metaplastic carcinomas may be enriched in epithelial to mesenchymal transition markers and in tumor-initiating cell specifically in the non-glandular elements. For this, we analyzed 27 primary metaplastic carcinomas and carried out a comprehensive protein expression analysis of the epithelial to mesenchymal transition regulators ZEB1 and E-cadherin and of tumor-initiating cells in light of tumor histopathology.

\section{Materials and methods}

\section{Tissue Samples}

A total of 27 metaplastic carcinomas were analyzed. All slides were obtained with Institutional Review Board approval from the surgical pathology files at the University of Michigan. Hematoxylin and eosinstained slides of formalin-fixed, paraffin-embedded tumors were reviewed and confirmed independently and blindly by two pathologists (YZ and CGK). Metaplastic carcinomas were classified and graded according to the 2003 WHO (World Health Organization) classification of metaplastic carcinomas. ${ }^{1}$ The metaplastic carcinomas were graded on the basis of the metaplastic component as grade 1 (low), grade 2 (intermediate) or grade 3 (high) following described criteria. ${ }^{1}$ In all cases, the diagnosis of metaplastic carcinoma was confirmed by positive cytokeratin staining including a cytokeratin cocktail (AE1/AE3, Cam5.2) and/or a highmolecular weight cytokeratin stain (34bE12). Clinical information and pathological features including patient age, tumor stage, hormonal receptor status and HER-2/neu overexpression, lymph node and distant metastasis were recorded from the surgical pathology reports.

\section{Immunohistochemistry and Scoring}

The expression of E-cadherin and ZEB1, and the expression of CD44 and CD24 was analyzed simultaneously by using double immunostaining. Aldehyde dehydrogenase-1 (ALDH-1) was determined by single immunohistochemistry. Briefly, paraffinembedded tissue blocks were sectioned at $5 \mu \mathrm{m}$ and placed on charged slides. Slides were deparaffinized in xylene and rehydrated through graded alcohols. Heat induced epitope retrieval was performed in the Decloaking Chamber (Biocare Medical) with target retrieval, pH 6.0 (DakoCytomation). Slides were incubated in 3\% hydrogen peroxide for $5 \mathrm{~min}$ to quench endogenous peroxidases. Double stain slides were incubated in Rodent Block M (Biocare Medical) for $30 \mathrm{~min}$ at room temperature. All slides were then incubated for $1.5 \mathrm{~h}$ at room temperature with either mouse monoclonal anti-ALDH-1 (BD Biosciences, Cat\# 611194) diluted 1:6000, or an antibody cocktail containing rabbit polyclonal anti-ZEB1 (Santa Cruz Biotechnology, Cat\# SC-25388) diluted 1:800 and mouse monoclonal anti-E-Cadherin (BD Biosciences, Cat\# 610182) diluted 1:5000), or an antibody cocktail containing rabbit monoclonal anti-CD44 (Abcam, Cat\# AB51037) diluted 1:500 and mouse monoclonal anti-CD24 (Biocare Medical, Cat\# CM323) diluted 1:120). Slides were incubated for $30 \mathrm{~min}$ at room temperature with either anti-mouse Envision ${ }^{+}$ horseradish peroxidase-labeled Polymer (DakoCytomation), Mach 2 Double Stain1 or Mach 2 Double Stain 2 (Biocare Medical), respectively. Horseradish peroxidase staining was visualized with the $\mathrm{DAB}^{+}$ Kit (DakoCytomation) and AP staining was visualized with Vulcan Fast Red (Biocare Medical). Negative control slides omitting primary antibodies were run. Slides were counterstained in hematoxylin, blued in running tap water, dehydrated through graded alcohols, clered in xylene and then mounted with Permount.

Expression of ZEB1 nuclear protein was categorized as positive (nuclear staining in $\geq 5 \%$ of tumor cells) or negative (no nuclear staining or nuclear staining in $<5 \%$ of tumor cells). ${ }^{18}$ E-cadherin staining was considered as normal when expressed at the cytoplasmic membranes with crisp complete pattern, or aberrant when membranous staining was reduced or absent, following previously validated studies. ${ }^{19}$ Tumors presenting at least one ALDH-1-positive, or CD44 ${ }^{+} / \mathrm{CD} 24^{- \text {llow }}$ cancer cell were considered positive following published studies and taking into account their biological significance. ${ }^{20-22}$

The association between the expression of ALDH-1, CD44 ${ }^{+} / \mathrm{CD} 24^{-/ \text {low }} \mathrm{ZEB} 1$, and E-cadherin proteins, their relationship to clinical and pathological characteristics, and their possible association with epithelial and/or mesenchymal differentiation in metaplastic carcinomas was analyzed by using 
X2-test and Fisher's exact test. A $P$-value of $<0.05$ was considered statistically significant.

\section{Results}

\section{Clinical and Histopathological Features of the Metaplastic Carcinomas}

The clinical and histological features of the metaplastic carcinomas are summarized in Table 1. All patients were female, with a median age of 60 years (range 33-87). Axillary lymph node metastases were identified in eight cases $(30 \%)$. All of the tumors with available information were negative for ER, PR and for HER-2/neu expression. Of the cases with available staging information, four tumors were Stage I, 11 Stage II, 2 Stage III and 3 Stage IV. Distant metastases were observed in four cases, including in the lung parenchyma, pleura, brain, and vertebrae.

Of the 27 metaplastic breast carcinomas $20(74 \%)$ had squamous and/or spindle sarcomatous areas and $7(26 \%)$ had heterologous elements (6 chondroid and 1 osseous). An adjacent glandular component was seen in $11(41 \%)$ tumors. Of the 7 spindle metaplastic carcinoma, none had histological grade 1, $4(57 \%)$ had grade 2 and $3(43 \%)$ had grade 3 . Three of the metaplastic carcinomas with

Table 1 Summary of clinical and pathologic information on the 27 patients with metaplastic carcinoma of the breast included in our study

\begin{tabular}{lc} 
Characteristic & \\
\hline No. of patients & 27 \\
Median age (range) & $60(36-87)$ \\
& \\
Pathologic stage, n (\%) & $15(56)$ \\
I/II & $5(19)$ \\
III/IV & $7(26)$ \\
Unknown & \\
Median tumor size (range), cm & $(0.5-10.5)$ \\
& \\
Lymph nodes, n (\%) & $14(52)$ \\
Negative & $8(30)$ \\
Positive & $5(19)$ \\
Unknown & \\
Site of distant metastasis, n & 2 \\
Lung and pleural & 1 \\
Vertebrae & 1 \\
Brain & \\
Predominant metaplastic component, n (\%) & $13(48)$ \\
Squamous & $7(26)$ \\
Spindle & $6(22)$ \\
Chondroid & $1(4)$ \\
Osseous & \\
Histologic grade, n (\%) & \\
1 & \\
2 & \\
3 & \\
\hline & \\
\hline & \\
& \\
&
\end{tabular}

squamous differentiation also showed spindle cell metaplasia, the other had only squamous elements. The squamous components ranged from moderate to poorly differentiated carcinoma. Those tumors with chondroid and osseous differentiation had features of chondrosarcoma and osteosarcoma, respectively. All glandular areas revealed moderate to poorly differentiated invasive ductal carcinoma.

\section{Metaplastic Carcinomas Express Tumor-Initiating Cell Markers in the Metaplastic Components}

Out of 27 metaplastic carcinoma cases, 17 (63\%) contained ALDH-1-positive cells. In most of these cases, the cytoplasmic staining was detected in groups of plump spindle-shaped cells as well as in the squamous nests (Figures $1 \mathrm{~b}, \mathrm{f}$ and $\mathrm{j}$ ). Further detailed analysis of each individual component revealed ALDH-1-positive cells in 16 out of 27 $(59 \%)$ metaplastic components, including in $71 \%$, $39 \%, 83 \%$, and $100 \%$ of the spindle, squamous, chondroid and osseous cells, respectively. In contrast, ALDH-1-positive cells were rare in the glandular component (present in 1 of 11 cases, 9\%; Fischer's exact test, $P=0.0017$, Figure $1 \mathrm{~b}$ arrow). There were no significant differences among the subtypes of metaplastic carcinoma for the expression of ALDH-1.

We found that cells expressing CD $44^{+} / \mathrm{CD} 24^{-/ \text {low }}$ were present in 12 out of $27(44 \%)$ metaplastic carcinomas. CD $44^{+} / \mathrm{CD} 24^{-/ \text {low }}$ cells were absent in the glandular elements and detected in 6 of 13 $(46 \%)$ tumors with squamous areas, in 5 of $7(71 \%)$ tumors with spindle areas and in 1 of $6(17 \%)$ of tumors with chondroid elements (Figure 1). CD $44^{+} /$ CD24 $4^{- \text {low }}$ cells could not be identified in the osseous component. The results of ALDH-1 and CD44/CD24 staining with respect to predominant metaplastic component and glandular areas are summarized in Table 2.

\section{Metaplastic Carcinomas Exhibit Frequent Loss of E-cadherin and Increased Expression of ZEB1, an Inducer of Epithelial to Mesenchymal Transition}

Simultaneous expression of ZEB1 and E-cadherin proteins in metaplastic carcinoma tissue samples was analyzed by dual-staining immunohistochemistry. This technique allowed us to determine the expression pattern and levels of each protein in the same cells. As expected, when present, E-cadherin protein was only expressed in the cytoplasmic membrane of epithelial cells whereas ZEB1 was expressed in the nucleus. We found a significant inverse association between these markers; the staining for E-cadherin protein was reduced or lost and ZEB1 protein expression was enhanced in the same tumor cells (Figure 1).

Of 27 metaplastic carcinomas, E-cadherin was aberrant (reduced or lost) in all mesenchymal 


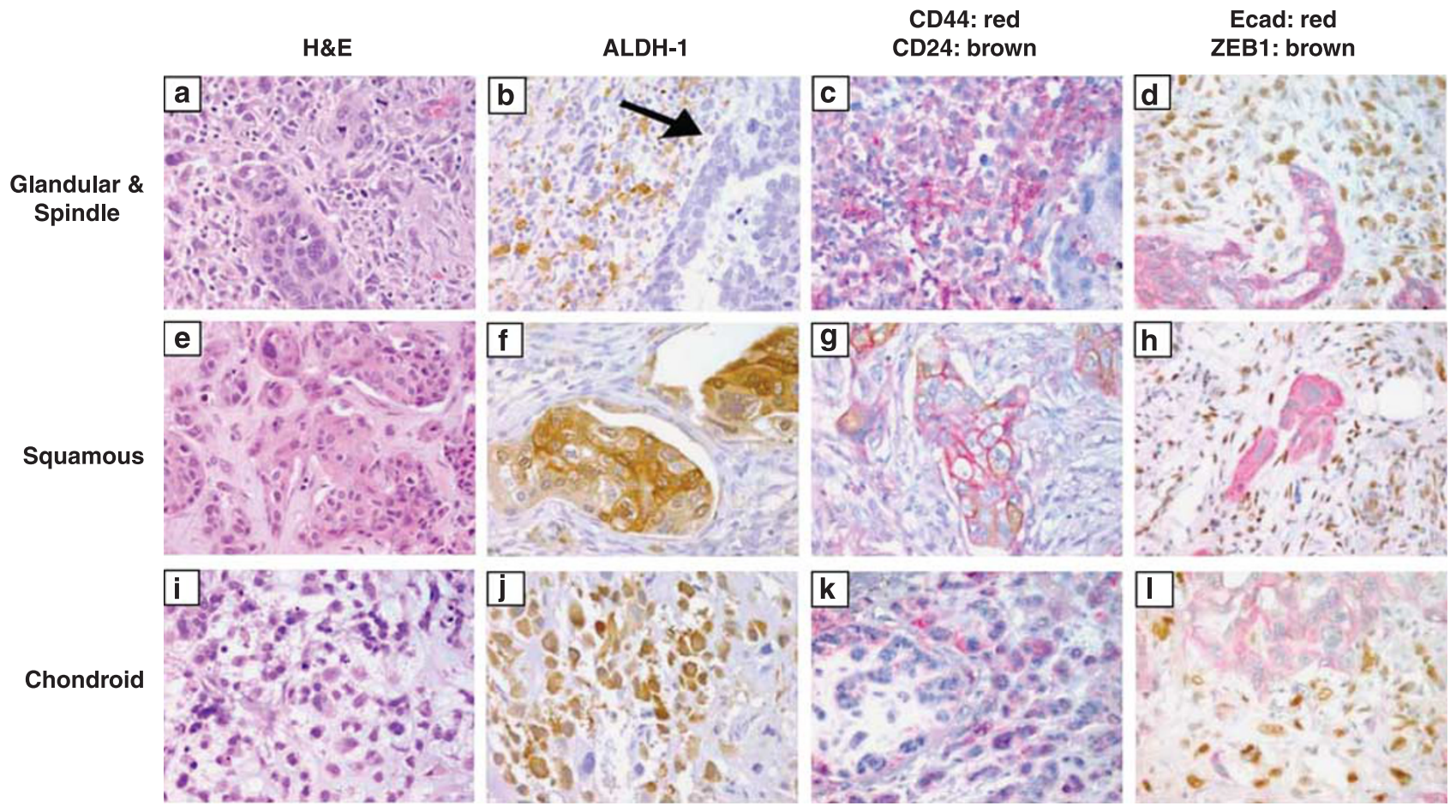

Figure 1 Immunohistochemical staining of ALDH-1, CD44/CD24, ZEB1 and E-cadherin in metaplastic carcinomas of the breast. (a) Metaplastic carcinoma with glandular and spindle cell differentiation; (b) same tumor showing expression of ALDH-1 in the spindle component. Arrow shows the absence of ALDH-1 expression in a malignant gland; (c) same tumor showing that the malignant spindle cells are positive for CD44 (red) and negative for CD24 (brown); (d) double immunostaining for ZEB1 and E-cadherin shows that the glands express normal E-cadherin but E-cadherin is downregulated in the spindle cells, which concomitantly upregulate ZEB1; (e-h) representative pictures of a metaplastic carcinoma with squamous differentiation; Note the high ALDH-1 reactivity in the squamous nests (f); (i-l) metaplastic carcinoma with chondroid differentiation showing ALDH-1 and CD44 expression in the chondroid areas, as well as upregulation of ZEB1 and decrease in E-cadherin expression. All pictures are $\times 400$ magnification. ALDH-1, aldehyde dehydrogenase-1.

Table 2 ALDH-1, CD44+ ${ }^{+}$CD24 ${ }^{- \text {low }}$, ZEB1 and E-cadherin expression in relationship to the predominant metaplastic component and glandular component

\begin{tabular}{|c|c|c|c|c|c|}
\hline \multirow[t]{2}{*}{ Component (n) } & \multirow[t]{2}{*}{$A L D H-1(\%)$} & \multirow[t]{2}{*}{$C D 44^{+} / C D 24^{-1 l o w}(\%)$} & \multirow[t]{2}{*}{$Z E B 1(\%)$} & \multicolumn{2}{|c|}{ E-cadherin } \\
\hline & & & & Normal & Aberrant \\
\hline Metaplastic component (27) & $16(59)$ & $12(44)$ & $11(41)$ & $13(48)$ & $14(52)$ \\
\hline Squamous (13) & $5(39)$ & $6(46)$ & 0 & $13(100)$ & 0 \\
\hline Spindle (7) & $5(71)$ & $5(71)$ & $6(86)$ & 0 & $7(100)$ \\
\hline Chondroid (6) & $5(83)$ & $1(17)$ & $5(83)$ & 0 & $6(100)$ \\
\hline Osseous (1) & $1(100)$ & 0 & $1(100)$ & 0 & $1(100)$ \\
\hline Glandular component (11) & $1(9)$ & 0 & 0 & $11(100)$ & 0 \\
\hline
\end{tabular}

Abbreviation: ALDH-1, aldehyde dehydrogenase-1.

metaplastic components. Specifically, E-cadherin was aberrant in cells differentiated toward spindle ( 7 of 7 cases), chondroid (6 of 6 cases) and osseous (1 of 1 case) components. On the other hand, E-cadherin expression level was normal in all the glands as well as in all (13 of 13 cases) squamous areas.

ZEB1 staining was detected in 41\% (11 out of 27) of metaplastic carcinomas. All the ZEB1-positive tumors exhibited mesenchymal differentiation toward spindle, chondroid or osseous. The glandular and squamous areas were negative for ZEB1 expression in all cases (Fischer's exact test $P=0.002$, Table 2 and Figure 1). We noted that in the transition from epithelial to mesenchymal elements there was a decrease of E-cadherin and an increase in ZEB1 expression (Figures 1d, $\mathrm{h}$ and $\mathrm{l}$ ). Details on the differential distribution of ZEB1 and E-cadherin staining with respect to predominant metaplastic component of each metaplastic carcinoma are summarized in Table 2. Aberrant E-cadherin, positive ZEB1 and the presence of ALDH-1 and 
CD $44^{+} / \mathrm{CD} 24^{-/ \text {low }}$ cells were significantly associated in the metaplastic areas $\left(\chi^{2}, P<0.05\right)$.

\section{Discussion}

Because breast carcinoma arises from glandular epithelium, it exhibits the features of adenocarcinoma. ${ }^{4}$ However, in some cases, part or all of the neoplastic cells show non-glandular growths by a process termed 'metaplasia,' which signifies the change of one cell type into another. Metaplasia is thought to proceed through reprogramming of pluripotent stem cells. ${ }^{4}$ This characterizes the special type of breast carcinoma termed metaplastic carcinoma. Although it remains to be determined whether metaplastic carcinomas arise from malignant transformation of particular cancer stem cells or through a process of dedifferentiation, ${ }^{23}$ cytogenetic and molecular studies suggest that the glandular and non-glandular components of these tumors originate from a common cell population. ${ }^{24,25} \mathrm{We}$ hypothesized that the phenotypic changes of metaplastic carcinomas in the breast are likely the result of alterations of genes involved in cell fate and differentiation.

Although metaplastic carcinomas of the breast are uncommon, they exhibit an aggressive behavior and poor clinical outcome. ${ }^{26}$ Metaplastic carcinomas are largely negative for hormone receptors and for HER2/neu overexpression. ${ }^{2,26}$ Recent studies have linked the process of epithelial to mesenchymal transition to the induction of stem cell features in breast cancer cells, ${ }^{8,9}$ and in particular in the metaplastic subtype. ${ }^{14,16}$ However, how these molecular findings relate to the heterogeneous histological features of metasplastic carcinomas has not been investigated, and thus remains incompletely understood. We undertook this study to investigate the presence of breast cancer cells expressing markers of stem cells, their relationship to the expression of epithelial to mesenchymal transition proteins and to specific histological components of metaplastic carcinomas.

We found that the non-glandular elements of metaplastic carcinomas express biological markers of an epithelial to mesenchymal transition. One of these markers, ZEB1, is transcriptional repressor of the E-cadherin gene in breast and other cells. ${ }^{10,27,28}$ Our lab has demonstrated that in breast epithelial cells, upregulation of ZEB1 leads to E-cadherin downregulation through direct binding to the E-cadherin proximal promoter. ${ }^{12}$ In this study, using double immunohistochemistry we detected the gradual loss of E-cadherin and acquisition of ZEB1 overexpressing mesenchymal-like cellular phenotype. These data are intriguing in light of our previous studies showing that metaplastic carcinomas harbor mutations in the Wnt-induced secreted protein-3 gene (WISP3/CCN6), ${ }^{29}$ which is a novel regulator of ZEB1 and E-cadherin expression in benign breast cells and in breast cancer cells. ${ }^{10}$
Taken together, these data pave the way to investigating the specific roles of ZEB1 and WISP3/CCN6 in the induction of mesenchymal-like non-glandular elements in metaplastic carcinoma, with potential therapeutic implications.

Although multiple putative breast cancer stem cell markers have been proposed, ALDH-1 and CD44 +/CD24-/low are the most studied and validated breast cancer stem cell marker proteins to date. ${ }^{21,30,31}$ ALDH-1 is a detoxifying enzyme responsible for the oxidation of intracellular aldehydes, which has been shown to identify and to promote breast cancer stem cell properties in vivo and in vitro. ${ }^{30,31}$ It is important to note that ALDH-1 immunostaining was found to be associated with estrogenand progesterone-negative tumors. ${ }^{31}$ We found that metaplastic carcinomas are enriched for ALDH-1-

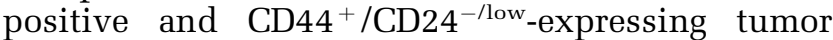
cells. Neither of these stem cell markers was expressed by the cancer cells forming glands, but rather by cancer cells in the metaplastic, nonglandular elements. Specifically, metaplastic carcinomas with squamous and/or spindle components contained ALDH-1 and $\mathrm{CD} 44^{+} / \mathrm{CD} 24^{- \text {low }}$ breast cancer cells. Even though we found a significant association between the stem cell marker expression in the non-glandular areas, ALDH-1 and CD44 ${ }^{+}$/

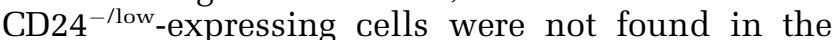
same tumor areas. These results suggest that these two markers may identify cells with different stem cell properties or cells at different stages of differentiation, and warrant further investigation.

Cytotoxic chemotherapy is the current recommended treatment for patients with metaplastic breast carcinomas. ${ }^{32,33}$ However, these tumors do not respond favorably to treatment, with common recurrences and poor prognosis. ${ }^{34-36}$ Our data demonstrate that metaplastic carcinomas of the breast contain cancer cells expressing stem cell markers ALDH-1 and the combination of CD44 ${ }^{+}$/

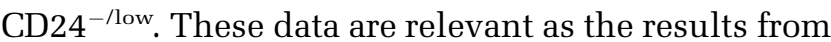
neoadjuvant clinical studies have led to propose the hypothesis that cancer stem cells are resistant to chemotherapy compared with the bulk tumor cell populations. ${ }^{37,38}$ Thus, patients with metaplastic carcinomas may constitute candidates to test novel therapies against breast cancer stem cells, already in clinical trials. ${ }^{39}$

In summary, our study shows that metaplastic carcinomas of the breast have histological evidence and express biological markers of an epithelial to mesenchymal transition characterized by progressive overexpression of ZEB1 and downregulation of E-cadherin. This change in morphology and protein expression pattern is associated with an acquisition of breast cancer stem cell marker proteins ALDH-1 and $\mathrm{CD} 44^{+} / \mathrm{CD} 24^{-/ \text {low}}$, which may have distinct functional roles in metaplastic carcinomas. As the process of epithelial to mesenchymal transition and the presence of breast cancer stem cells have been reported to enhance breast cancer metastasis, our 
data support the hypothesis that blockade of the epithelial to mesenchymal transition and/or tumor stem cells may improve the outcome of patients with metaplastic breast carcinomas.

\section{Acknowledgements}

This work was supported by the NIH grants R01 CA107469, R01 CA125577 and U01CA154224 (CGK), the Fashion Charitable Foundation of New York/QVC, and the National Institutes of Health through the University of Michigan's Cancer Center Support Grant (5 P30 CA46592).

\section{Disclosure/conflict of interest}

The authors declare no conflict of interest.

\section{References}

1 Tavassoli FA, Devilee P. Pathology and Genetics of Tumors of the Breast and Female Genital Organs. IARC Press: Lyon, 2003.

2 Reis-Filho JS, Milanezi F, Steele D, et al. Metaplastic breast carcinomas are basal-like tumours. Histopathology 2006;49:10-21.

3 Lacroix-Triki M, Geyer FC, Lambros MB, et al. betacatenin/Wnt signalling pathway in fibromatosis, metaplastic carcinomas and phyllodes tumours of the breast. Mod Pathol 2010;23:1438-1448.

4 Kumar V, Fausto N, Aster J. Robbins and Cotran Pathologic Basis of Disease 8th edn. Elsevier: Philadelphia, PA, 2010.

5 Hugo H, Ackland ML, Blick T, et al. Epithelialmesenchymal and mesenchymal-epithelial transitions in carcinoma progression. J Cell Physiol 2007;213:374-383.

6 Peinado H, Olmeda D, Cano A. Snail, Zeb and bHLH factors in tumour progression: an alliance against the epithelial phenotype? Nat Rev Cancer 2007;7:415-428.

7 Zhou BP, Deng J, Xia W, et al. Dual regulation of Snail by GSK-3beta-mediated phosphorylation in control of epithelial-mesenchymal transition. Nat Cell Biol 2004;6:931-940.

8 Mani SA, Guo W, Liao MJ, et al. The epithelialmesenchymal transition generates cells with properties of stem cells. Cell 2008;133:704-715.

9 Blick T, Hugo H, Widodo E, et al. Epithelial mesenchymal transition traits in human breast cancer cell lines parallel the CD44(hi/)CD24 (lo/-) stem cell phenotype in human breast cancer. J Mammary Gland Biol Neoplasia 2010;15:235-252.

10 Lorenzatti G, Huang W, Pal A, et al. CCN6 (WISP3) decreases ZEB1-mediated EMT and invasion by attenuation of IGF-1 receptor signaling in breast cancer. J Cell Sci 2011;124:1752-1758.

11 Aigner K, Dampier B, Descovich L, et al. The transcription factor ZEB1 (deltaEF1) promotes tumour cell dedifferentiation by repressing master regulators of epithelial polarity. Oncogene 2007;26:6979-6988.

12 Huang W, Zhang Y, Varambally S, et al. Inhibition of CCN6 (Wnt-1-induced signaling protein 3) down- regulates E-cadherin in the breast epithelium through induction of snail and ZEB1. Am J Pathol 2008;172: 893-904.

13 Schmalhofer O, Brabletz S, Brabletz T. E-cadherin, beta-catenin, and ZEB1 in malignant progression of cancer. Cancer Metastasis Rev 2009;28:151-166.

14 Hennessy BT, Gonzalez-Angulo AM, Stemke-Hale K, et al. Characterization of a naturally occurring breast cancer subset enriched in epithelial-to-mesenchymal transition and stem cell characteristics. Cancer Res 2009;69:4116-4124.

15 Creighton CJ, Chang JC, Rosen JM. Epithelial-mesenchymal transition (EMT) in tumor-initiating cells and its clinical implications in breast cancer. J Mammary Gland Biol Neoplasia 2010;15:253-260.

16 Taube JH, Herschkowitz JI, Komurov K, et al. Core epithelial-to-mesenchymal transition interactome gene-expression signature is associated with claudinlow and metaplastic breast cancer subtypes. Proc Natl Acad Sci USA 2010;107:15449-15454.

17 Lien HC, Hsiao YH, Lin YS, et al. Molecular signatures of metaplastic carcinoma of the breast by large-scale transcriptional profiling: identification of genes potentially related to epithelial-mesenchymal transition. Oncogene 2007;26:7859-7871.

18 Singh M, Spoelstra NS, Jean A, et al. ZEB1 expression in type I vs type II endometrial cancers: a marker of aggressive disease. Mod Pathol 2008;21:912-923.

19 Kowalski PJ, Rubin MA, Kleer CG. E-cadherin expression in primary carcinomas of the breast and its distant metastases. Breast Cancer Res 2003;5: R217-R222.

20 Kunju LP, Cookingham C, Toy KA, et al. EZH2 and ALDH-1 mark breast epithelium at risk for breast cancer development. Mod Pathol 2011;24:786-793.

21 Al-Hajj M, Wicha MS, Benito-Hernandez A, et al. Prospective identification of tumorigenic breast cancer cells. Proc Natl Acad Sci USA 2003;100: 3983-3988.

22 Abraham BK, Fritz P, McClellan M, et al. Prevalence of CD44+/CD24-/low cells in breast cancer may not be associated with clinical outcome but may favor distant metastasis. Clin Cancer Res 2005;11:1154-1159.

23 van Deurzen CH, Lee AH, Gill MS, et al. Metaplastic breast carcinoma: tumour histogenesis or dedifferentiation? J Pathol 2011;224:434-437.

24 Teixeira MR, Qvist H, Bohler PJ, et al. Cytogenetic analysis shows that carcinosarcomas of the breast are of monoclonal origin. Genes Chromosomes Cancer 1998;22:145-151.

25 Geyer FC, Weigelt B, Natrajan R, et al. Molecular analysis reveals a genetic basis for the phenotypic diversity of metaplastic breast carcinomas. J Pathol 2010;220:562-573.

26 Rosen PP. Rosen's breast pathology In: Rosen PP (ed). Rosen's Breast Pathology Third edn. Lippincott Williams \& Wilkins: Philadelphia, PA, 2008.

27 Eger A, Aigner K, Sonderegger S, et al. DeltaEF1 is a transcriptional repressor of E-cadherin and regulates epithelial plasticity in breast cancer cells. Oncogene 2005;24:2375-2385.

28 Graham TR, Zhau HE, Odero-Marah VA, et al. Insulinlike growth factor-I-dependent up-regulation of ZEB1 drives epithelial-to-mesenchymal transition in human prostate cancer cells. Cancer Res 2008;68:2479-2488.

29 Hayes MJ, Thomas D, Emmons A, et al. Genetic changes of Wnt pathway genes are common events in 
metaplastic carcinomas of the breast. Clin Cancer Res 2008;14:4038-4044.

30 Dontu G, Abdallah WM, Foley JM, et al. In vitro propagation and transcriptional profiling of human mammary stem/progenitor cells. Genes Dev 2003;17: 1253-1270.

31 Ginestier C, Hur MH, Charafe-Jauffret E, et al. ALDH1 is a marker of normal and malignant human mammary stem cells and a predictor of poor clinical outcome. Cell Stem Cell 2007;1:555-567.

32 Lester TR, Hunt KK, Nayeemuddin KM, GonzalezAngulo AM, Feig BW, et al. Metaplastic sarcomatoid carcinoma of the breast appears more aggressive than other triple receptor-negative breast cancers. Breast Cancer Res Treat 2011; e-pub ahead of print.

33 Carlson RW, Allred DC, Anderson BO, et al. Breast cancer. Clinical practice guidelines in oncology. J Natl Compr Canc Netw 2009;7:122-192.
34 Rayson D, Adjei AA, Suman VJ, et al. Metaplastic breast cancer: prognosis and response to systemic therapy. Ann Oncol 1999;10:413-419.

35 Oberman HA. Metaplastic carcinoma of the breast. A clinicopathologic study of 29 patients. Am J Surg Pathol 1987;11:918-929.

36 Hennessy BT, Giordano S, Broglio K, et al. Biphasic metaplastic sarcomatoid carcinoma of the breast. Ann Oncol 2006;17:605-613.

37 Kakarala M, Wicha MS. Implications of the cancer stem-cell hypothesis for breast cancer prevention and therapy. J Clin Oncol 2008;26:2813-2820.

$38 \mathrm{Li} \mathrm{X}$, Lewis MT, Huang J, et al. Intrinsic resistance of tumorigenic breast cancer cells to chemotherapy. J Natl Cancer Inst 2008;100:672-679.

39 Korkaya H, Liu S, Wicha MS. Regulation of cancer stem cells by cytokine networks: attacking cancers inflammatory roots. Clin Cancer Res 2011;17:6125-6129. 